\title{
THE ROLE OF STARS IN THE ENERGETICS OF LINERs
}

\author{
Dan Maoz
}

School of Physics and Astronomy, Tel-Aviv University, Tel-Aviv 69978, Israel

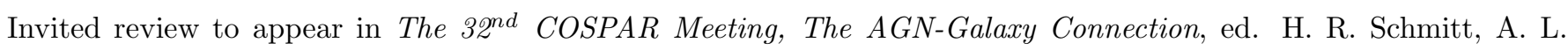
Kinney, and L. C. Ho (Advances in Space Research).

\begin{abstract}
Imaging studies have shown that $\sim 25 \%$ of LINER galaxies display a compact nuclear UV source. I compare the HST ultraviolet (1150-3200 $\AA$ ) spectra that are now available for seven such "UVbright" LINERs. The spectra of NGC 404, NGC 4569, and NGC 5055 show clear absorption-line signatures of massive stars, indicating a stellar origin for the UV continuum. Similar features are probably present in NGC 6500. The same stellar signatures may be present but undetectable in NGC 4594, due to the low signal-to-noise ratio of the spectrum, and in M81 and NGC 4579, due to superposed strong, broad emission lines. The compact central UV continuum source that is observed in these galaxies is a nuclear star cluster rather than a low-luminosity active galactic nucleus (AGN), at least in some cases. At least four of the LINERs suffer from an ionizing photon deficit, in the sense that the ionizing photon flux inferred from the observed far-UV continuum is insufficient to drive the optical H I recombination lines. Examination of the nuclear X-ray flux of each galaxy shows a high X-ray/UV ratio in the four "UV-photon starved" LINERs. In these four objects, a separate component, emitting predominantly in the extreme-UV, is the likely ionizing agent, and is perhaps unrelated to the observed nuclear UV emission. Future observations can determine whether the UV continuum in LINERs is always dominated by a starburst or, alternatively, that there are two types of UV-bright LINERs: starburst-dominated and AGN-dominated. Interestingly, recent results show that starbursts dominate the nuclear energetics in many Seyfert $2 \mathrm{~s}$ as well.
\end{abstract}

\section{INTRODUCTION}

Low-ionization nuclear emission-line regions (LINERs) are detected in the nuclei of a large fraction of all bright nearby galaxies (Ho, Filippenko, \& Sargent 1997a; Ho, this volume). Since their definition as a class by Heckman (1980), they have elicited debate as to their nature and relation, if any, to active galactic nuclei (AGNs). On the one hand, the luminosities of most LINERs are unimpressive compared to "classical" AGNs, and can easily be produced by processes other than accretion onto massive black holes. Indeed, LINER-like spectra are sometimes seen to arise in "non-nuclear" environments, such as cooling flows. On the other hand, a variety of observables point to similarities and continuities between AGNs and at least some LINERs (see Ho, this volume). If LINERs represent the low-luminosity end of the AGN phenomenon, then they are the nearest and most common ex- 
amples, and their study is germane to understanding AGN demographics, quasar evolution, dormant black holes in quiescent galaxies, the X-ray background, and the connection between AGN-like and starburst-like activity.

The ultraviolet (UV) sensitivity and angular resolution of the Hubble Space Telescope (HST) is providing new clues toward understanding LINERs. Maoz et al. (1996a) carried out a UV (2300 A) imaging survey of the central regions of 110 nearby galaxies with the Faint Object Camera (FOC) on HST. As reported in Maoz et al. (1995), five among the 25 LINERs in their sample revealed nuclear UV sources, in most cases unresolved, implying physical sizes $\lesssim 2$ pc. Maoz et al. (1995) argued that the UV sources in these "UV-bright" LINERs could be the extension of the ionizing continuum, which is rarely seen in LINERs at optical wavelengths due to the strong background from the normal bulge population. The compactness of the sources suggested that they could be nonstellar in nature, although compact star clusters of such luminosity were also possible. A similar fraction of UV-bright nuclei was found in a $2200 \AA$ imaging survey of 20 LINER and low-luminosity Seyfert 2 galaxies carried out with the WFPC2 camera on HST by Barth et al. (1996a; 1998). A compact, isolated, nuclear UV source has also been found in FOC images of the LINER NGC 4594 at 3400 $\AA$ by Crane et al. (1993), and in WFPC2 images of the LINER M81 in a broad (1100 $\AA$ to $2100 \AA)$ bandpass by Devereux, Ford, \& Jacoby (1997). The UV-bright LINERs were obvious targets for follow-up spectroscopy with HST . Faint Object Spectrograph (FOS) observations have been analyzed for the LINERs M81 (Ho, Filippenko, \& Sargent 1996), NGC 4579 (Barth et al. 1996b), NGC 6500 (Barth et al. 1997), and NGC 4594 (Nicholson et al. 1998).

The spectra of M81 and NGC 4579, which in the optical range have weak broad wings in the $\mathrm{H} \alpha$ line (Filippenko \& Sargent 1985; 1988; Ho et al. 1997b), are reminiscent of AGNs in the UV, with strong, broad emission lines superposed on a featureless continuum. As discussed by Barth et al. (1996b), in NGC 4579 the FOS $2200 \AA$ flux is less than $1 / 3$ that implied by the FOC measurement made 19 months earlier, implying variability of a nonstellar continuum source. It would be valuable, however, to confirm such variability in this and other LINERs. Ho et al. (1996) and Barth et al. (1996b) concluded that these two LINERs are most probably AGNs. NGC 6500 and NGC 4594, on the other hand, have only weak and narrow emission lines on top of a UV continuum. Based on the resolved appearance of the UV source in the HST WFPC2 F218W image, and the tentative detection of optical Wolf-Rayet features, Barth et al. (1997) concluded that the UV emission in NGC 6500 is likely dominated by massive stars, though contribution from a scattered AGN component could not be excluded. For NGC 4594, Nicholson et al. (1998) favor an AGN interpretation. Maoz et al. (1998) presented HST spectra for three additional UV-bright LINERs (NGC 404, NGC 4569, and NGC 5055), and compared the properties of all seven UV-bright LINERs observed spectroscopically with HST to date. I summarize below the results of that analysis.

\section{FAR-UV SPECTRAL SIGNATURES OF MASSIVE STARS}

Figure 1 shows most of the G130H spectrum of all seven LINERs. The objects are ordered with the two broad-lined LINERs (M81 and NGC 4579) on top, and the other LINERs in order of decreasing $f_{\lambda}$. Figure 2 shows in more detail the same spectral region for NGC 4569, NGC 404, NGC 5055, and NGC 6500. Overlayed on each spectrum (thin line) is a scaled, normalized version of the HST GHRS spectrum of the "B" clump in NGC 1741, a starburst galaxy, which is described by Conti, Leitherer \& Vacca (1996). The broad, blueshifted absorption profiles of C IV $\lambda 1549$ and Si IV $\lambda 1400$ (and also N V $\lambda 1240$ ) in NGC 1741-B are the signatures of winds produced by massive stars (see Leitherer, Robert, \& Heckman 1995). The other, narrower, absorption lines are of both photospheric and interstellar-medium (ISM) origin. The characteristic starburst features seen in the spectrum of 


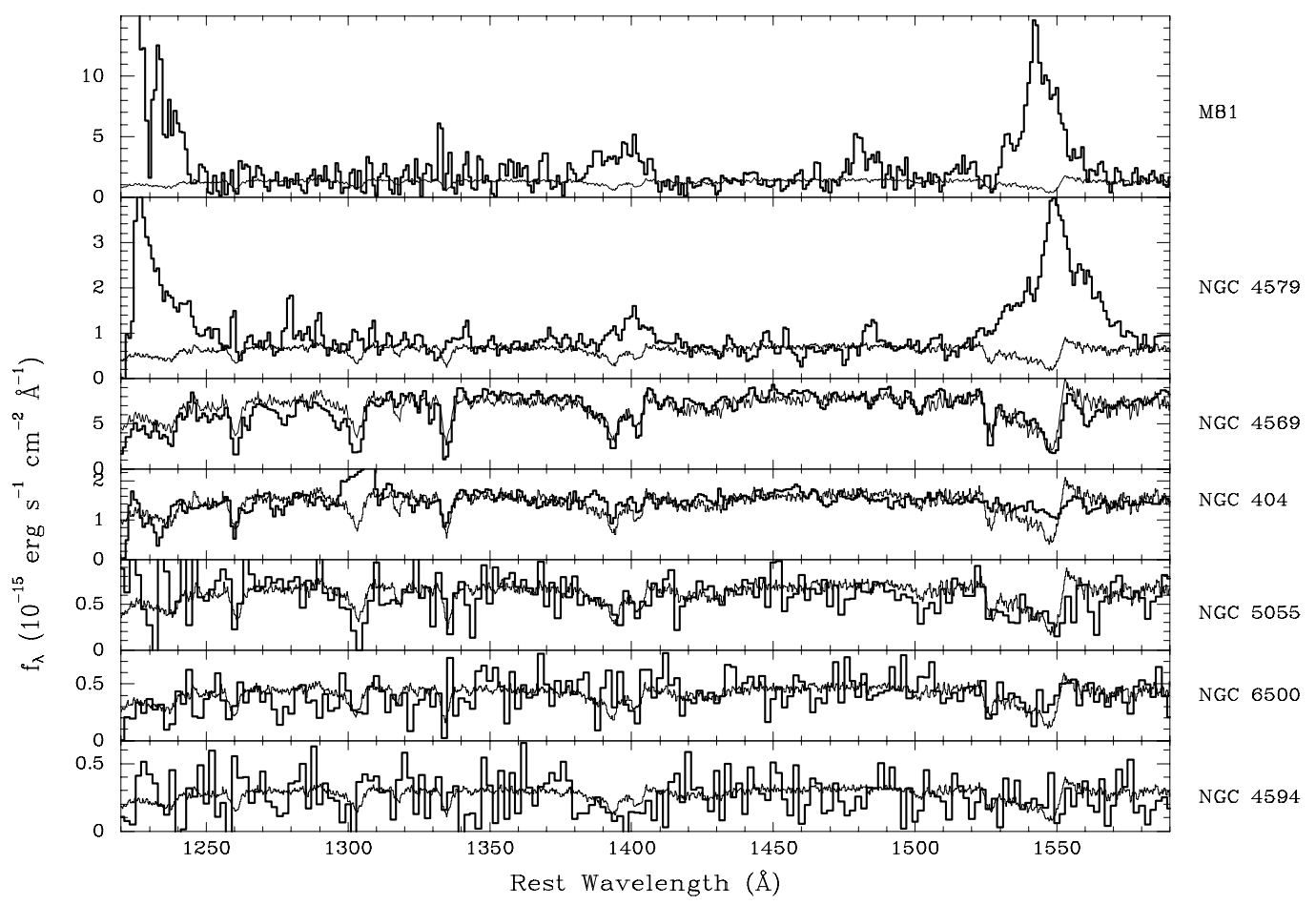

Fig. 1: FOS G130H spectra of the seven LINERs (bold lines), ordered with the two broad-lined objects on top, and then with decreasing $f_{\lambda}$. Overlayed in each case is the spectrum of the starburst in NGC 1741-B, normalized to be flat in $f_{\lambda}$ and scaled by a multiplicative factor to match the LINER continuum level.

NGC 1741-B are also observed in star-forming regions in 30 Doradus (Vacca et al. 1995) and NGC 4214 (Leitherer et al. 1996), in star-forming galaxies at redshifts $z \approx 2-3$ (Steidel et al. 1996; Lowenthal et al. 1997), and in the $z=3.8$ starburst radio galaxy 4C 41.17 (Dey et al. 1997).

The FOS spectrum of NGC 4569, the brightest (and highest S/N) LINER in our sample, is virtually identical to that of NGC 1741-B. Most of the features in the two spectra match one-to-one. NGC 4569 has all the main interstellar absorption lines seen in starburst spectra, as well as the broad stellar wind features. Also detected are several narrow absorption lines which cannot be interstellar (they are not resonance lines) and constitute further evidence for the photospheres of hot stars: Si III $\lambda \lambda 1294 / 1297$, Si III $\lambda 1417$, C III $\lambda 1427, \mathrm{~S}$ V $\lambda 1502$, and N IV $\lambda 1720$ (Heckman \& Leitherer 1997). The correspondence between the two spectra is such that a dilution of the stellar features by a nonstellar (i.e., featureless) continuum producing more than $20 \%$ of the UV continuum would be readily apparent. The only significant difference between the spectra of the starburst clump in NGC 1741 and the nucleus of NGC 4569 is the presence of relatively strong C I absorption lines (at rest wavelengths $1277 \AA, 1280 \AA, 1329 \AA, 1561 \AA$, and $1657 \AA$ ) in NGC 4569.円 All the absorption lines are at the slight blueshift $\left(-223 \mathrm{~km} \mathrm{~s}^{-1}\right)$ of the galaxy, and hence are not produced in the Milky Way's ISM. There is no doubt that the UV emission in this object, even though its source is highly compact ( $\lesssim 2$ pc; Maoz et al. 1995), is dominated by a cluster of massive stars. The luminosity of a star cluster is dominated by the most massive stars. Using spectral synthesis models and assuming a

1 The C I lines are generally not seen with such strength (up to $3.5 \AA$ equivalent width) in starburst spectra. C I has a low ionization potential $(11.26 \mathrm{eV})$, so it can form only in places that are shielded from UV radiation by dust, at the interfaces of molecular clouds. Deeper inside the clouds the carbon gets bound in CO. Interestingly, the same C I lines seem to be present in some of the other LINERs as well, so they may be a signature of a particular sort of environment. 


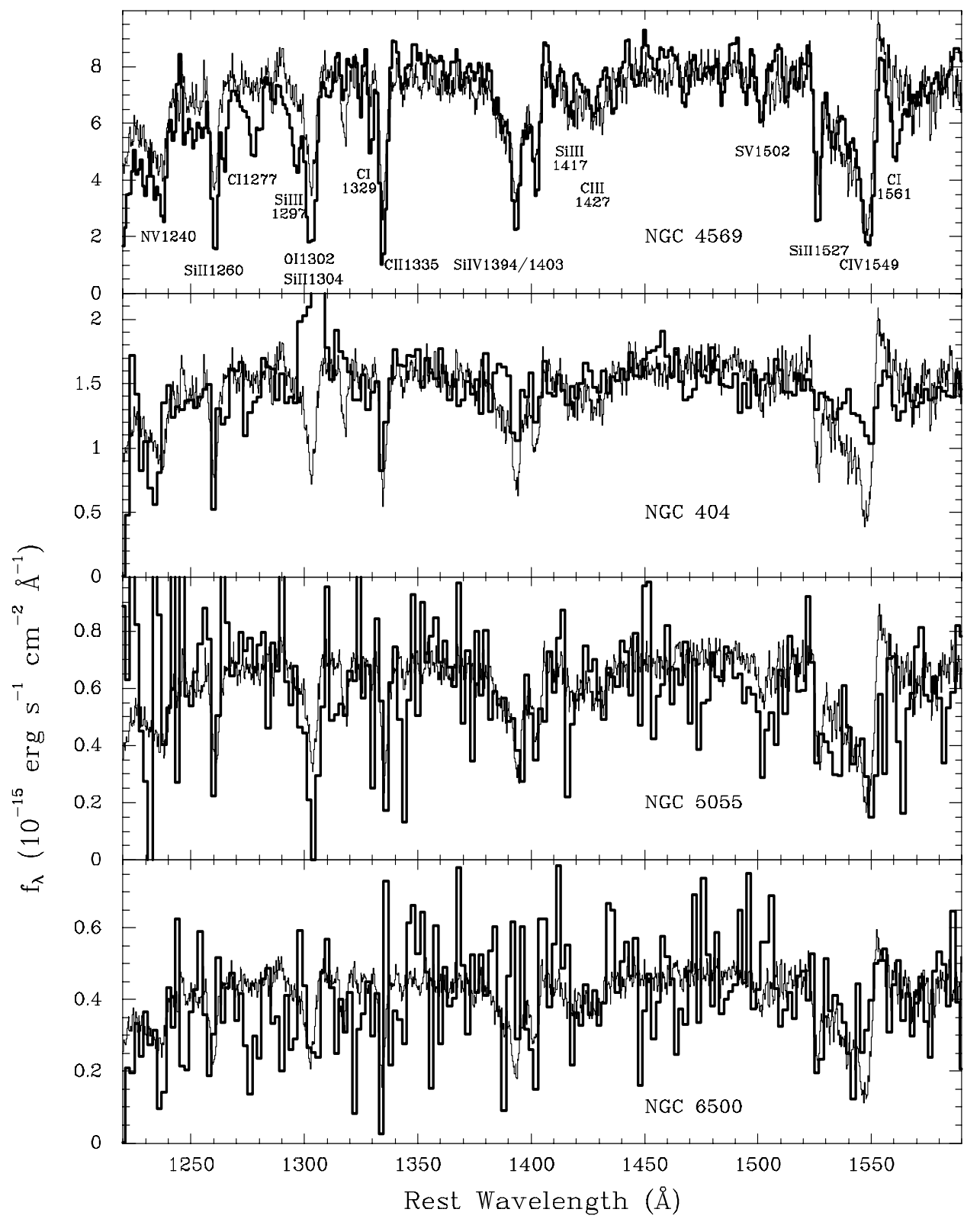

Fig. 2: Same as Figure 1, but only for the four brightest LINERs devoid of broad emission lines. The strongest absorption lines are marked for NGC 4569. The emission feature near $1300 \AA$ in NGC 404 is an artifact. 
distance of $9 \mathrm{Mpc}$, we estimate that 250-600 O-type stars (depending on the presence of very massive early-O-type stars) are sufficient to produce the observed luminosity. A plausible amount of dust extinction can raise this number by factors of up to several tens.

Proceeding to the far-UV spectrum of NGC 404 (Fig. 2), we note that the broad, blueshifted C IV absorption is shallower, but definitely detected. Si IV absorption is not seen, except in the narrow components. According to the synthesis by Leitherer et al. (1995), such spectra characterize a starburst that is either very young $(<3 \mathrm{Myr})$ or $>5 \mathrm{Myr}$ old. The denser winds of O-type supergiants, which are present only during a limited time, are required in order to produce the broad blueshifted Si IV absorption. The C IV absorption, on the other hand, remains as long as there are main-sequence O-type stars. The relative shallowness of the $\mathrm{C}$ IV profile can also be reproduced well by diluting the NGC 1741-B starburst spectrum by a featureless (e.g. nonstellar) spectrum that is constant in $f_{\lambda}$, and contributes $\sim 60 \%$ of the total flux. The strongest interstellar absorption lines that appear in NGC 4569, including the C I lines, are seen in NGC 404 as well. The velocity width and blueshift of the C IV profile clearly point to a stellar origin for this line. (The "emission" between $1295 \AA$ and $1310 \AA$ in NGC 404 is an artifact due to a noisy diode that masks the O I and S II absorptions that are probably present at those wavelengths.) It thus appears that the UV continuum source in this LINER is a star cluster that is of different age than the one in NGC 4569, or perhaps a cluster of similar age whose emission is diluted by a nonstellar continuum. At a $2 \mathrm{Mpc}$ distance to NGC 404, its UV luminosity is 100 times lower than that of NGC 4569, implying just two to six O-type stars can produce the observed luminosity. Again, the actual number is likely higher after a reasonable extinction correction.

Continuing to the G130H spectrum of NGC 5055 (Fig. 2), the S/N degrades, but the blueshifted broad C IV and Si IV absorptions seen in NGC 4569 are definitely present. Their minima are at the galaxy's redshift (497 $\mathrm{km} \mathrm{s}^{-1}$ ). The stellar nature of this UV source is not surprising, since Maoz et al. (1995) already noted that it is marginally resolved at $2200 \AA$, with $\mathrm{FWHM} \approx 0.2^{\prime \prime}(\approx 6 \mathrm{pc})$. NGC 4569 and NGC 5055 are remarkably similar over the entire UV range. Overall, the spectrum of NGC 5055 resembles a lower S/N version of that of NGC 4569. In the spectrum of NGC 6500 (Fig. 2 ), the blueshifted broad C IV absorption is possibly recognizable although, as noted by Barth et al. (1997), its significance is arguable when the spectrum is viewed individually. The comparison to the spectra of the other LINERs and the starburst spectrum, combined with the obvious degradation in $\mathrm{S} / \mathrm{N}$ in this fainter object, suggest that the absorption may, in fact, be present. The spectrum is too noisy and dominated by scattered light to reach any conclusion regarding the Si IV absorption. As noted above, Barth et al. (1997) already concluded, based on the extended $\left(0.5^{\prime \prime} \approx 100 \mathrm{pc}\right)$ appearance of the UV source in a WFPC2 image, that this source is probably stellar in nature. Finally, the NGC 4594 G130H data (Fig. 1) are too noisy and dominated by scattered light to reach any conclusion, except that they could be consistent with the same type of spectrum. There are certainly no strong emission lines in this part of the spectrum of NGC 4594.

We conclude that, in the five LINERs without emission lines in the G130H range, the UV continuum is, with varying degrees of certainty, produced by massive, young stars. Could the same be true of the two broad-lined LINERs in our sample, M81 and NGC 4579? As already noted by Ho et al. (1996) for M81, one cannot answer this question based on these UV data alone. Figure 1 shows that if the continuum spectrum were of the same starburst type as in the other LINERs, we would not know it because the broad emission lines are coincident with the broad absorptions. A case in point is the recent study by Heckman et al. (1997) of the Seyfert 2 galaxy Mrk 477. The relatively narrow emission lines are superposed on the broad stellar-wind signatures, but in Si IV and N V enough of the stellar absorptions are visible near the blue wings of the emission lines to reveal the starburst 
nature of the UV continuum emission. In M81 and NGC 4579, however, the emission lines are too broad to see the stellar absorptions, if they are there. The single argument that the UV continuum source in NGC 4579 is necessarily nonstellar is its possible factor $\sim 3$ variability reported in Barth et al. (1996b).

Barring perhaps NGC 4579 (assuming its UV variability is real), the UV continuum in all the LINERs could be stellar in origin. Note that this result does not bear directly on the question of whether or not there is also a nonstellar, quasar-like object in the nucleus. A microquasar could still be present, and dominate the emission at wavelengths other than the UV. Furthermore, it is becoming increasingly appreciated that circumnuclear starbursts, albeit on physical scales larger than those considered here, can contribute significantly to the UV and optical brightness of some AGNs (e.g., IC 5135 - Shields \& Filippenko 1990; Heckman et al. 1998; NGC 1068 - Thatte et al. 1997; NGC 7469 - Genzel et al. 1995; Mrk 477 - Heckman et al. 1997; some Seyfert 2s - Gonzalez-Delgado et al. 1998).

\section{THE IONIZING PHOTON BUDGET}

We have compiled $\mathrm{H} \alpha$ fluxes for each object from Ho et al. (1997a), Stauffer (1982; NGC 5055), and Keel (1983; NGC 4569). Figure 3 (left panel) shows the $\mathrm{H} \alpha$ flux vs. $f_{\lambda}(1300 \AA)$ measured from the spectra and corrected for Galactic extinction. There appears to be little relation between these observables. We have computed the ratio of the $\mathrm{H} \alpha$ line flux to the the $1300 \AA$ continuum flux density for young star clusters containing populations of O-type stars. Further details of these and related computations are described in Maoz et al. (1998) and Sternberg (1998). Here we consider young clusters ( $\lesssim 10^{6}$ yr old) with Salpeter initial-mass functions (IMFs). Assuming case-B recombination in $10^{4} \mathrm{~K}$ ionization-bounded nebulae, the $\mathrm{H} \alpha$ line luminosities per number of O-type stars are equal to $1.4 \times 10^{36}$ and $1.4 \times 10^{37} \mathrm{erg} \mathrm{s}^{-1}$ for IMFs which extend up to $30 M_{\odot}$ and $120 M_{\odot}$, respectively. For such clusters the $\mathrm{H} \alpha / f_{\lambda}(1300 \AA)$ ratios equal about 10.5 and $42.0 \AA$, and decrease with increasing cluster age. The stellar-wind signatures in several of the LINERs require the presence of stars of mass $\gtrsim 30 M_{\odot}$. The two solid diagonal lines in Figure 3 show the maximum $\mathrm{H} \alpha$ flux that can be produced with $100 \%$ covering factor from ionization by a stellar population with the given $1300 \AA$ flux and upper mass cutoff of $120 M_{\odot}$ or $30 M_{\odot}$. The two dotted lines show this limit for ionization by power-law continua of the form $\lambda^{\beta}$ with $\beta=-1$ or $\beta=0$.

We see that, if there is no internal extinction, then some and possibly all of the LINERs require an additional ionizing source to drive their line flux. M81, NGC 4594, and NGC 6500, even if their UV spectra are extrapolated as power laws with $\beta=-1$ (i.e., harder than observed) rather than as stellar-population spectra, also have a severe ionizing photon deficit. NGC 4579, on the other hand, does have a $\beta \approx-1$ slope. If the Maoz et al. (1995) flux level is adopted, then as before, it has a factor 2.8 ionizing photon surplus, rather than a deficit. If, however, stars dominate the UV continuum or the FOS flux level is the "normal" level, then this LINER also has an ionizing photon deficit. In the three LINERs whose UV emission is clearly dominated by stars (NGC 404, NGC 4569, and NGC 5055), ionization by the stellar population can provide the required power, but only if very massive stars are still present. Interestingly, it is these three objects which also have the lowest emission-line ratios of [O III] $\lambda 5007 / \mathrm{H} \beta,[\mathrm{O} \mathrm{I}] \lambda 6300 / \mathrm{H} \alpha$, and $[\mathrm{S} \mathrm{II}] \lambda \lambda 6716,6731 / \mathrm{H} \alpha$ in the sample (see Ho et al. 1997a); this is exactly what one would expect from ionization by the relatively soft continuum of a significant stellar component, which produces less heating per ionization and a smaller partially ionized zone than an AGN-like power-law continuum.

Alternatively, some internal foreground extinction (such that the UV emission is attenuated as seen by 

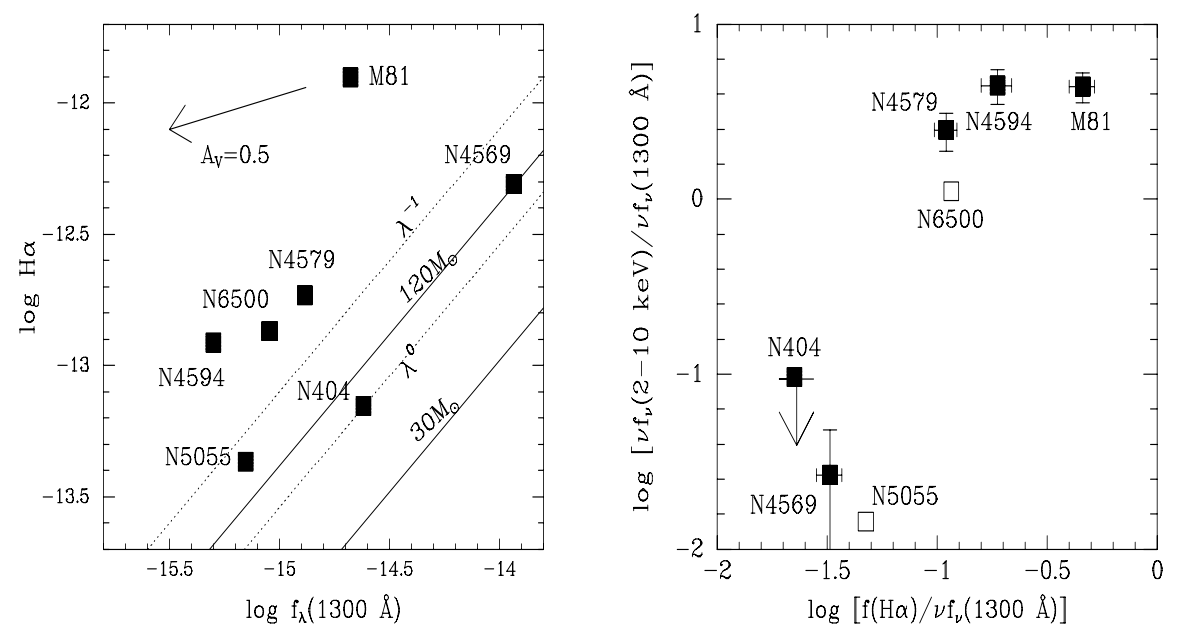

Fig3 : Left: Log H $\alpha$ flux (in erg s $\mathrm{s}^{-1} \mathrm{~cm}^{-2}$ ) vs. $\log f_{\lambda}\left(1300 \AA\right.$ ) (in $\operatorname{erg~s}^{-1} \mathrm{~cm}^{-2} \AA^{-1}$ ) measured from the spectra and corrected for Galactic extinction. The two solid diagonal lines show the maximum $\mathrm{H} \alpha$ flux that can be produced in Case B recombination with $100 \%$ covering factor from ionization by a stellar population with a given $1300 \AA$ flux, resulting from an instantaneous burst of age 1 Myr, Salpeter initial-mass function, and upper mass cutoff of $120 M_{\odot}$ or $30 M_{\odot}$. The two dotted lines show this limit for ionization by power-law continua $f_{\lambda} \propto \lambda^{\beta}$ with $\beta=-1$ or $\beta=0$. An $A_{V}=0.5 \mathrm{mag}$ foreground extinction vector, assuming a Galactic extinction curve, is shown for reference. Typical uncertainties are $10 \%$ in $f_{\lambda}(1300 \AA)( \pm 0.04$ in the $\log )$ and $30 \%$ in $\mathrm{H} \alpha$ flux $(\sim \pm 0.13$ in the $\log )$. Right: The ratio of X-ray-to-UV power vs. H $\alpha$-to-UV power for each of the LINERs. Filled symbols denote $A S C A$ measurements, empty symbols are extrapolated ROSAT values. Note how in the four "UV-photon starved" objects (high $\mathrm{H} \alpha$-to-UV power ratio) the X-ray power is comparable to, or greater than, the UV power. An energy source emitting primarily in the X-rays, and not necessarily related to the observed UV source, is probably the main ionizing agent in the four "UV-photon starved" objects. 
the observer, but not as seen by the ionized gas) could, in principle, alleviate the ionization budget problem in some of the objects. The uncertainty in the extinction curve and the many possible configurations for different extinction of the continuum and nebular emission result in a broad range of possible extinction corrections. To illustrate the effect of a plausible extinction correction, Figure 3 shows an $A_{V}=0.5 \mathrm{mag}$ foreground extinction vector, assuming a Galactic extinction curve. A Galactic curve is intermediate in "greyness" to the SMC and Calzetti et al. (1994) curves. Note that the objects with the most severe ionizing photon deficits (M81, NGC 4594, and NGC 6500) are those whose Balmer decrements indicate little internal reddening, $A_{V}=0.1$ to $0.35 \mathrm{mag}$, for the range in extinction curves. In NGC 4569, NGC 5055, and NGC 4579, a plausible extinction correction will increase the intrinsic $1300 \AA$ flux by a factor $\gtrsim 10$ (with a corresponding increase in the number of O-type stars $)$, and may relieve the need for the most massive $\left(\sim 120 M_{\odot}\right)$ stars.

We conclude that at least some of the LINERs in our sample have an ionizing photon deficit, indicating an additional energy source, beyond that implied by the observable UV. To search for evidence for such an additional source, we have compiled X-ray data for the seven LINERs. For NGC 404, NGC 4569, NGC 4579, and NGC 4594 we have used analysis of both archival and new $A S C A$ data by Y. Terashima, A. Ptak, and L. Ho (see Terashima, this volume) to obtain unabsorbed 2-10 keV fluxes. For M81 we have used the $A S C A$ flux derived by Ishisaki et al. (1996). NGC 5055 and NGC 6500 have not been observed with $A S C A$, but have been observed by ROSAT in the 0.1-2.4 keV band. We have extrapolated these measurements to the $A S C A$ bandpass, as described in Maoz et al. (1998), though of course such extrapolations are highly uncertain and should be treated with caution.

Figure 3 (right panel) shows the ratio of X-ray-to-UV power vs. H $\alpha$-to-UV power for each of the LINERs. Note how in the four "UV-photon starved" objects (high $\mathrm{H} \alpha$ to UV power ratio) the X-ray power is comparable to or greater than the UV power. Conversely, in the three objects without a serious ionizing photon deficit, the X-ray power is one to two orders of magnitude lower than the UV power. This suggests that, indeed, an energy source emitting primarily in the extreme-UV, and not directly related to the observed UV source, may be the main ionizing agent in the four "UV-photon starved" objects. The observed X-ray emission would be the high-energy tail of such a component. For example, a blackbody with temperature $>3.3 \times 10^{5} \mathrm{~K}$ would have an X-ray to UV power ratio greater than or equal to that of the UV-photon starved objects. Alternatively, the photoionizing continua in these objects could consist of power-law spectra extending from the X-rays to the Lyman limit. However, this would require that the central UV sources are significantly attenuated by dust extinction while the nebular components are not.

It has long been debated whether the emission lines in LINERs are produced by means of photoionization or shocks. The question has been recently re-addressed in the analysis of the four LINERs among the seven discussed here with published UV spectra (Ho et al. 1996; Barth et al. 1996b, 1997; Nicholson et al. 1998). These studies show that the UV line ratios are consistent with either photoionization by an AGN-like continuum or by slow-moving shocks, but inconsistent with the fast "photoionizing" shocks proposed by Dopita \& Sutherland (1996). The data discussed here show that massive stars exist in some LINERs. The sources required to produce most or all of the emission lines by photoionization are therefore present, at least sometimes. 


\section{CONCLUSIONS}

The HST UV spectra of seven LINERs having compact nuclear UV sources reveal the following. 1. At least three of the LINERs have clear spectral signatures indicating that the dominant UV continuum source is a cluster of massive stars.

2. A similar continuum source could dominate in the other four LINERs as well, but its spectral signatures would be veiled by low $\mathrm{S} / \mathrm{N}$ or superposed broad emission lines. Alternatively, there may be two types of UV-bright LINERs: those where the UV continuum is produced by a starburst, and those where it is nonstellar. If the variability of NGC 4579 is real, its continuum source is certainly an AGN.

3. The three "clearly-stellar" LINERs have relatively weak X-ray emission, and their stellar populations probably provide enough ionizing photons to explain the observed optical emission-line flux. The four other LINERs have severe ionizing photon deficits, for reasonable extrapolations of their UV spectra beyond the Lyman limit, but an X-ray/UV power ratio that is higher by two orders of magnitudes than that of the three stellar LINERs. A component which emits primarily in the extreme-UV may be the main photoionizing agent in these four objects.

The picture emerging from this comparison is that the compact UV continuum source seen in $\sim 25 \%$ of LINERs (Maoz et al. 1995; Barth et al. 1996a, 1998) is, at least in some cases, a nuclear starburst rather than an AGN-like nonstellar object. The UV luminosity is driven by tens to thousands of O-type stars, depending on the object and the extinction assumed. The O-stars could be the highmass end of a bound stellar population, similar to those seen in super star clusters (e.g., Maoz et al. 1996b) . Nonstellar sources in LINERs may be significant or even dominate at other wavelengths, as we indeed find for some of the objects. Even the three "clearly-stellar" LINERs, which do not obviously require the existence of such an additional source, may well have one; the ionizing photon budget estimate was made assuming a 100\% covering factor of the line-emitting gas, which is not necessarily true. This picture fits well with recent results showing that nuclear-starburst and quasarlike activity are often intermingled in Seyfert 1 and 2 galaxies. For example, Gonzalez-Delgado et al. (1998) show that the so-called "featureless continuum" that has been previously observed in some Seyfert $2 \mathrm{~s}$ is not really featureless, and is due to massive stars. Their preliminary analysis of the 20 brightest Seyfert 2s indicates that massive stars are present in at least one-third of the cases. Our results extend this result to the lower luminosities of the LINERs discussed here, although the question of whether a "micro-quasar" exists in these objects is still open.

Higher S/N UV spectra of some of the objects in our sample could reveal whether they too have the signatures of massive stars. Conversely, further evidence of UV continuum variability, as suggested in NGC 4579, should be sought in LINERs. Even in those LINERs with stellar signatures, UV variability could reveal a contribution by a nonstellar component. This work suggests, however, that the AGNs possibly associated with LINERs are most prominent at higher energies. X-ray observations by upcoming space missions, having better angular and spectral resolution and higher sensitivity, will likely provide key insights to the nature of LINERs.

\section{REFERENCES}

Barth, A.J., Ho, L.C., Filippenko, A.V., \& Sargent, W.L.W. 1996a, in "The Physics of LINERs in View of Recent Observations", eds. M. Eracleous et al. (San Francisco: ASP), p. 153 Barth, A.J, Reichert, G.A., Filippenko, A.V., Ho, L.C., Shields, J.C., Mushotzky, R.F. \& Puchnarewicz, E.M. 1996b, AJ, 112, 1829 
Barth, A.J, Reichert, G.A., Ho, L.C., Shields, J.C., Filippenko, A.V., \& Puchnarewicz, E.M. 1997, AJ, 114, 2313

Barth, A.J., Ho, L.C., Filippenko, A.V., \& Sargent, W.L.W. 1998, ApJ, 496, 133

Calzetti, D., Kinney, A.L., \& Storchi-Bergmann, T. 1994, ApJ, 429, 582

Conti, P.S., Leitherer, C., \& Vacca, W.D. 1996, ApJ, 461, L87

Devereux, N., Ford, H., \& Jacoby, G. 1997, ApJ, 481, L71

Dey, A., van Breugel, W., Vacca, W.D., \& Antonucci, R. 1997, ApJ, 490, 698

Filippenko, A. V., \& Sargent, W. L. W. 1985, ApJS, 57, 503

Filippenko, A. V., \& Sargent, W. L. W. 1988, ApJ , 324, 134

Gonzalez-Delgado, R.M., et al. 1998, ApJ, in press

Heckman, T.M. 1980, A\&A, 87, 152

Heckman, T.M., \& Leitherer, C. 1997, AJ, 114, 69

Heckman, T.M., et al. 1997, ApJ, 482, 114

Ho, L.C., Filippenko, A.V., \& Sargent, W.L.W. 1996, ApJ, 462, 183

Ho, L. C., Filippenko, A. V., \& Sargent, W. L. W. 1997a, ApJS, 112, 315

Ho, L. C., Filippenko, A. V., Sargent, W. L. W., \& Peng, C. Y. 1997b , ApJS, 112, 391

Keel, W.C. 1983, ApJ, 269, 466

Leitherer, C., \& Heckman, T.M. 1995, ApJS, 96, 9

Leitherer, C., Vacca, W.D., Conti, P.S., Filippenko, A.V., Robert, C., \& Sargent, W.L.W. 1996, ApJ, 465, 717

Leitherer, C., Robert, C., \& Heckman, T.M. 1995, ApJS, 99, 173

Lowenthal, J.D., et al. 1997, ApJ, 481, 673

Maoz, D., Barth, A.J., Sternberg, A., Filippenko, A.V., Ho, L.C., Macchetto, F.D., Rix, H.-W., \& Schneider, D.P. 1996b, AJ, 111, 2248

Maoz, D., Filippenko, A.V., Ho, L.C., Macchetto, F.D., Rix, H.-W., \& Schneider, D.P. 1996a, ApJS, 107, 215

Maoz, D., Filippenko, A. V., Ho, L. C., Rix, H. -W., Bahcall, J. N., Schneider, D. P., \& Macchetto, F. D. 1995, ApJ, 440, 91

Maoz, D., Koratkar, A. P., Shields, J. C., Ho, L. C., Filippenko, A. V., \& Sternberg, A. 1998, AJ, 116, 55

Nicholson, K.L., Reichert, G.A., Mason , K.O., Puchnarewicz, E.M., Ho, L.C., Shields, J.C., \& Filippenko, A.V. 1998, MNRAS, submitted

Shields, J. C., \& Filippenko, A. V. 1990, AJ, 100, 1034

Stauffer, J.R. 1982, ApJ, 262, 66

Steidel, C.C., Giavalisco, M., Dickinson, M., \& Adelberger, K.L. 1996, AJ, 112, 352

Sternberg, A. 1998, ApJ, in press

Thatte, N., Quirrenbach, A., Genzel, R., Maiolino R., \& Tecza, M. 1997, ApJ, 490, 238

Vacca, W.D., Robert, C., Leitherer, C., \& Conti, P.S. 1995, ApJ, 444, 647

\section{ACKNOWLEDGMENTS}

I thank the organizers for a stimulating and enjoyable meeting. 